\title{
Water Evaporation Optimization Algorithm for Solving Dynamic Economic Dispatch
}

\author{
R. Venkadesh ${ }^{1}$ and R. Anandhakumar ${ }^{2}$ \\ ${ }^{I}$ Department of Electrical Engineering, Annamalai University, Chidambaram, Tamil Nadu, India \\ ${ }^{2}$ Department of Electrical Engineering, Annamalai University, Chidambaram, Tamil Nadu, India
}

\begin{abstract}
This paper presents an efficient Water Evaporation Optimization algorithm (WEO) is proposed to solve a Dynamic Economic Dispatch problem (DED). The dynamic dispatch problem differs from the static economic dispatch problem by incorporating generator spinning reserve, ramp rate limits, and valve point loading and transmission losses. The proposed water evaporation optimization algorithm is based on the evaporation of a tiny amount of water molecules on the solid surfaces with different wettability which can be studied by molecular dynamics simulations. The performance of the WEO algorithm is tested on five unit system with spinning reserve and ten unit systems. The comparison of the simulation results prove that the proposed WEO algorithm have a better performance than the existing methods.
\end{abstract}

Keywords: Dynamic economic dispatch, water evaporation algorithm, molecular dynamics simulation, reserve constrain, ramp rate, valve-point loading effect.

\section{Introduction}

Dynamic Economic Dispatch (DED) is used to resolve the optimal generation schedule of on-line generators, so as to meet the predicted load demand over certain problem period of time at minimum operating cost under different system and operational constraints. The dynamic optimization problem may need to consider the spinning reserve requirements $\left(\mathrm{SRR}_{\mathrm{s}}\right)$ in order to incorporate the unit coupling of ramp rates at the unit level. Due to the ramp-rate constraints of a generator, the operational decision at an hour may affect the operational decision at a later hour. Traditionally valve point loading effects of the turbine were ignored and a convex quadratic fuel cost function was considered for the thermal units. However, a more realistic model must take into account the valve-point effects. It has a look-ahead ability which is necessary to schedule the load early so that the system can predict rapid load changes in near future. The DED problem can be formulated as a largescale, optimization problem, which is quite difficult due to its intrinsic high dimensional, non-convex and nonlinear nature. The dimension of the problem increases rapidly with the system size and the scheduling horizon [1].

Several optimization methods including classical and heuristic algorithms were applied to solve DED problem. The conventional methods consist of Linear Programming (LP) [2], Non-Linear Programming (NLP) [3], Quadratic Programming (QP) [4], Lagrangian Programming (LP) [5] and Mixed Integer Quadratic Programming (MIQP) [6]. The main drawbacks of these methods are only need to run in linear problem and not applicable to large scale system. Unfortunately, DED with non smooth or non convex cost functions in valve point loading can fail to get global optimal solutions.

To overcome this deficiency, turn to various heuristic techniques such as Genetic Algorithm (GA) [7], Simulated Annealing (SA) [8], Artificial Immune System (AIS) [9], Evolutionary Programming (EP) [10], Differential Evolution (DE) [11], Harmony Search (HS) [12], Artificial Bee Colony (ABC) [13], Imperialist Competitive Algorithm (ICA) [14], Seeker Optimization Algorithm (SOA) [15], Teaching Learning Algorithm (TLA) [16], Improved Particle Swarm Optimization (IPSO) [17], Chaotic Differential Evolution (IDE) [18], Modified Teaching Learning Algorithm (MTLA) [19], Self-Adaptive Modified Firefly Algorithm (SAMFO) [20], Improve Pattern Search (IPS) [21], Enhanced Cross Entropy (ECE) [25], Adaptive Particle Swarm Optimization (APSO) [28], Enhanced Bee Swarm Optimization (EBSO) [35], Deterministic Guided Particle Swarm Optimization (DGPSO) [37]. The main drawback of these heuristic techniques gives the results but struck the local minima and lack of guarantee of convergence infinite time for large scale DED problems.

Hybrid techniques are used to solve the DED problem, such as Hybrid Immune-Genetic Algorithm (IGA) [22], hybridization of Artificial Immune System and Sequential Quadratic Programming (AIS-SQP) [23], modified hybrid Evolutionary Programming-Sequential Quadratic Programming (EP-SQP) [24], Hybrid Differential Evaluation (HDE) [29], Chaotic Differential Bee Colony Optimization (CDBCO) [30], Improved Chaotic Particle Swarm Optimization (ICPSO) [31], Chaotic Self Adaptive Particle Swarm Optimization (CSAPSO) [32], Enhanced Adaptive Particle Swarm Optimization (EAPSO) [33], Time Varying Acceleration Coefficient - Improved Particle Swarm Optimization (TVAC-IPSO) [34], Adaptive Hybrid Differential Evolution (AHDE) [38], hybrid methods are consuming more time to compute the results. Because the structure 
of this methods are more complicated. Moreover, an appropriate integration point of two algorithms is very difficult to determine.

Recently, crisscross optimization (CSO) [26], Hybrid Quantum Particle Swarm Optimization (HQPSO) [36], Hybrid Immune Genetic Algorithm (HI-GA) [39] method is solving the DED problem. But the convergence stagnancy phenomena are very large to compute the result. The large system is not suitable for maintain the population of personal best solutions so as to greatly accelerate the convergence.

Newly, motivated by the shallow water theory, researchers have proposed Water Evaporation Optimization (WEO) algorithm for solving global optimization problem [27]. The WEO algorithm is conceptually simple and easy to implement. The WEO algorithmic search consists of both global and local search. This guarantees that the proposed algorithm is competitive with other efficient well-known metaheuristics. The objective of this papers it to use WEO algorithm to obtain the optimal dispatches and compare the performances in terms of quality of solution with the recent reports.

The rest of this paper is organized as follows. Section II details the ELD problem formulation. Brief description of WEO algorithm and implementation of WEO for solving ELD problem are presented in Section III. The comparison of numerical simulation results and discussion with recent literature results are detailed in Section IV. Conclusions are presented in Section V followed by references.

\subsection{Objective Function}

\section{Problem Formulation}

The main objective of DED problem is to economically assign the power output over the operating perspective while fulfilling the demand, unit constrains and minimizes the total fuel cost.

The objective of DED is the total fuel cost equation as formulated as:

$$
F_{i}\left(P_{i}\right)=\sum_{i=1}^{T} \sum_{i=1}^{N} a_{i} P_{i, t}{ }^{2}+b_{i} P_{i, t}+c_{i}+\left|e_{i} \times \sin \left(f_{i} \times\left(P_{i, \text { min }}-P_{i, t}\right)\right)\right|
$$

Where $\mathrm{F}_{\mathrm{i}}$ is the cost function of the $\mathrm{i}^{\text {th }}$ generator at time t. at, $\mathrm{P}_{\mathrm{i}}$ is the real power generated by the $\mathrm{i}^{\text {th }}$ generator, $\mathrm{N}$ is the total number of online participating generating units, and $a_{i}, b_{i}, c_{i}$ is the cost coefficients of $i^{\text {th }}$ generator. $e_{i}$ and $f_{i}$ are the cost coefficients of $i^{\text {th }}$ generator reflecting valve point loading effects and $P_{i, \text { min }}$ is the minimum output power of $\mathrm{i}^{\text {th }}$ generating unit. $\mathrm{T}$ is the total number of hours in the scheduling horizon.

\subsection{Constraints}

\subsubsection{Power Balance}

The total power generation must satisfy sum of the demand and losses.

$$
\begin{aligned}
& P_{D, t}+P_{L, t}=\sum_{i=1}^{N} P_{i, t} \\
& P_{L, t}=\sum_{i=1}^{T} \sum_{j=1}^{N} P_{i, t} B_{i j} P_{j, t}+\sum_{i=1}^{N} B_{o i} P_{i, t}+B_{o o}
\end{aligned}
$$

Where $P_{D}$ is the total load, $P_{L}$ is the transmission loss, $B_{i j}, B_{o i}, B_{o o}$ are the transmission loss coefficients.

\subsubsection{Generator Power Limit}

The generated power should be within its minimum and maximum limits.

$$
P_{i, \text { min }} \leq P_{i} \leq P_{i, \max }
$$

$\mathrm{P}_{\mathrm{i}, \min }$ and $\mathrm{P}_{\mathrm{i}, \text { max }}$ is the minimum and maximum output power of $\mathrm{i}^{\text {th }}$ generating unit.

\subsubsection{Ramp Rate Limit}

To avoid undue thermal stresses on the boiler and the combustion equipment, the rate of change of the output power of each thermal unit must not exceed a certain ramp limit rate during increasing or decreasing the power output of each unit. This can be mathematically as follows.

$$
\max \left(P_{i, \text { min }}, P_{i, t}^{0}-D R_{i}\right) \leq P_{i, t} \leq \min \left(P_{i, \max }, P_{i, t}^{0}+U R_{i}\right)
$$

Such that max $\left(\mathrm{P}_{\mathrm{i} \text { min }}, \mathrm{P}_{\mathrm{i}, \mathrm{t}}{ }^{0}-\mathrm{DR}_{\mathrm{i}}\right)=\mathrm{P}_{\mathrm{i}, \mathrm{tmin}}$ and $\min \left(\mathrm{P}_{\mathrm{i} \max }, \mathrm{P}_{\mathrm{i}, t}{ }^{0}+\mathrm{UR}_{\mathrm{i}}\right)=\mathrm{P}_{\mathrm{i}, \mathrm{t} \max }$ where $\mathrm{P}_{\mathrm{i}, \mathrm{t} \min }$ and $\mathrm{P}_{\mathrm{i}, t \max }$ are the minimum and maximum limit of the real power of the $\mathrm{i}^{\text {th }}$ unit at the $\mathrm{t}^{\text {th }}$ interval in $\mathrm{MW}$ and $\mathrm{P}_{\mathrm{i}, t}{ }^{0}$ is the power generated by the $\mathrm{i}^{\text {th }}$ unit at the $(\mathrm{t}-1)^{\text {th }}$ hour. $\mathrm{UR}_{\mathrm{i}}$ and $\mathrm{DR}_{\mathrm{i}}$ are the UP and DOWN ramp rate limits of the $\mathrm{i}^{\text {th }}$ unit in $\mathrm{MW} / \mathrm{h}$ 


\subsubsection{Spinning Reserve Requirements}

The $\mathrm{SRR}_{\mathrm{s}}$ should be measured as an additional constraint to stay away from an unpredicted large load to the system or a breakdown in a certain large unit. Hence $S_{R R}$ for the RCDED problem are formulated in three different ways.

$$
\begin{aligned}
& \left(\Delta_{T}^{(1)}=\sum_{i=1}^{N U} P_{i}^{\max }-\left(P_{D}+P_{\text {loss }}+S R_{T}\right) \geq 0\right) \\
& \left(\Delta_{T}^{(2)}=\sum_{i=1}^{N U}\left(\min \left(P_{i}^{\mathrm{max}}-P_{i, t}, U R_{i}\right)\right)-S R_{T}\right) \geq 0 \\
& \left(\Delta_{T}^{(3)}=\sum_{i=1}^{N U}\left(\min \left(P_{i}^{\mathrm{max}}-P_{i, t}, \frac{U R_{i}}{6}\right)\right)-S R_{T}^{\prime}\right) \geq 0
\end{aligned}
$$

Equations (6) and (7) are generally applied the DED problems within 60 min of being required. Equation (8) will exactly satisfy the 10 min of being required and its amount is related to the ramp up rate constraint of generating unit. For time interval of the ramp up rate of unit $\mathrm{i}$ is $\mathrm{UR}_{\mathrm{i}}(\mathrm{MW} / \mathrm{h})$, the equivalent amount for $10 \mathrm{~min}$ is $\mathrm{UR}_{\mathrm{i}} / 6$.

\section{Water Evaporation Optimization}

The evaporation of water is very important in biological and environmental science. The water evaporation from bulk surface such as a lake or a river is different from evaporation of water restricted on the surface of solid materials. In this WEO algorithm water molecules are considered as algorithm individuals. Solid surface or substrate with variable wettability is reflected as the search space. Decreasing the surface wettability (substrate changed from hydrophility to hydrophobicity) reforms the water aggregation from a monolayer to a sessile droplet. Such a behavior is consistent with how the layout of individuals changes to each other as the algorithm progresses. And the decreasing wettability of surface can represent the decrease of objective function for a minimizing optimization problem. Evaporation flux rate of the water molecules is considered as the most appropriate measure for updating individuals which its pattern of change is in good agreement with the local and global search ability of the algorithm and make this algorithm have well converged behavior and simple algorithmic structure. The details of the water evaporation optimization algorithm are well presented in [27].

In the WEO algorithm, each cycle of the search consists of following three steps (i) Monolayer Evaporation Phase, this phase is considered as the global search ability of the algorithm (ii) Droplet Evaporation Phase, this phase can be considered as the local search ability of the algorithm and (iii) Updating Water Molecules, the updating mechanism of individuals.

\subsection{Monolayer Evaporation Phase}

In the monolayer evaporation phase the objective function of the each individuals $\mathrm{Fit}_{\mathrm{i}}{ }^{\mathrm{t}}$ is scaled to the interval $[-3.5,-0.5]$ and represented by the corresponding $\mathrm{E}_{\text {sub }}(\mathrm{i})^{\mathrm{t}}$ inserted to each individual (substrate energy vector), via the following scaling function.

$$
E_{\text {sub }}(i)^{t}=\frac{\left(E_{\max }-E_{\min }\right) \times\left(F_{i t}^{t}-\operatorname{Min}(\text { Fit })\right)}{(\operatorname{MaX}(\text { Fit })-\operatorname{Min}(\text { Fit }))}+E_{\text {min }}
$$

where $E_{\max }$ and $E_{\min }$ are the maximum and minimum values of $E_{\text {sub }}$ respectively. After generating the substrate energy vector, the Monolayer Evaporation Matrix (MEP) is constructed by the following equation.

$$
M E P_{i j}^{t}= \begin{cases}1 & \text { if } \operatorname{rand}_{i j} \leq \exp \left(E_{\text {sub }}(i)^{t}\right) \\ 0 & \text { if } \text { rand }_{i j} \geq \exp \left(E_{\text {sub }}(i)^{t}\right)\end{cases}
$$

where $\mathrm{MEP}_{\mathrm{t}}^{\mathrm{ij}}$ isthe updating probability for the $\mathrm{j}^{\text {th }}$ variable of the $\mathrm{i}^{\text {th }}$ individual or water molecule in the $\mathrm{t}^{\text {th }}$ iteration of the algorithm. In this way an individual with better objective function is more likely to remain unchanged in the search space.

\subsection{Droplet Evaporation Phase}

In the droplet evaporation phase, the evaporation flux is calculated by the following equation. 
$J(\theta)=J_{o} P_{o}\left(\frac{2}{3}+\frac{\cos ^{3} \theta}{3}-\cos \theta\right)(1-\cos \theta)$

where $J_{o}$ and $P_{o}$ are constant values. The evaporation flux value is depends upon the contact angle $\Theta$, whenever this angle is greater and as a result will have less evaporation. The contact angle vector is represented the following scaling function.

$$
\theta(i)^{t}=\frac{\left(\theta_{\max }-\theta_{\min }\right) \times\left(F_{i t}^{t}-\operatorname{Min}(\text { Fit })\right)}{(\operatorname{Max}(F i t)-\operatorname{Min}(\text { Fit }))}+\theta_{\text {min }}
$$

where the min and max are the minimum and maximum functions. The $\Theta_{\min } \& \Theta_{\max }$ values are chosen between $-50^{\circ}<\Theta<-20^{\circ}$ is quite suitable for WEO. After generating contact angle vector $\Theta(\mathrm{i})^{\mathrm{t}}$ the Droplet Probability Matrix (DEP) is constructed by the following equation.

$$
D E P_{i j}^{t}= \begin{cases}1 & \text { if } \operatorname{rand}_{i j}<J\left(\theta_{i}^{(t)}\right) \\ 0 & \text { if } \operatorname{rand}_{i j} \geq J\left(\theta_{i}^{(t)}\right)\end{cases}
$$

where $\mathrm{DEP}_{\mathrm{ij}}^{\mathrm{t}}$ is the updating probability for the $\mathrm{j}^{\text {th }}$ variable of the $\mathrm{i}^{\text {th }}$ individual or water molecule in the $\mathrm{t}^{\text {th }}$ iteration of the algorithm.

\subsection{Updating Water Molecules}

In the WEO algorithm the number of algorithm individuals or number of water molecules (nWM) is considered constant in all $\mathrm{t}^{\text {th }}$ iterations, where $\mathrm{t}$ is the number of current iterations. Considering a maximum value for algorithm iterations $\left(t_{\max }\right)$ is essential for this algorithm to determine the evaporation phase and for stopping criterion. When a water molecule is evaporated it should be renewed. Updating or evaporation of the current water molecules is made with the aim of improving objective function. The best strategy for regenerating the evaporated water molecules is using the current set of water molecules $\left(\mathrm{WM}^{(\mathrm{t})}\right)$. In this way a random permutation based step size can be considered for possible modification of individual as:

$$
S=\operatorname{rand} .\left(W M^{(t)}[\text { permutel }(i)(j)]-W M^{(t)}[\text { permute } 2(i)(j)]\right)
$$

where rand is a random number in [0,1] range, permuteland permute 2 are different rows of permutation functions. $i$ is the number of water molecule, $j$ is the number of dimensions of the problem. The next set of molecules $\left(\mathrm{WM}^{(\mathrm{t}+1)}\right)$ is generated by adding this random permutation based step size multiplied by the corresponding updating probability (monolayer evaporation and droplet evaporation probability) and can be stated mathematically as:

$$
W M^{(t+1)}=W M^{(t)}+S \times\left\{\begin{array}{l}
M E P^{(t)} t \leq t_{\text {max }} / 2 \\
D E P^{(t)} t>t_{\text {max }} / 2
\end{array}\right.
$$

Each water molecule is compared and replaced by the corresponding renewed molecule based on objective function. It should be noted that random permutation based step size can help in two aspects. In the first phase, water molecules are more far from each other than the second phase. In this way the generated permutation based step size will guarantee global and local capability in each phase.

The WEO algorithm can be summarized as follows:

Step1: Initialize all the algorithm and problem parameters, randomly initialize all water molecules.

Step2: Generating water evaporation matrix

Every water molecule follow the evaporation probability rules specified for each phase of the algorithm based on the Eqs. (10) and (13). For $\mathrm{t} \leq \mathrm{t}_{\max } / 2$, water molecules are globally evaporated based on monolayer evaporation probability MEP by using Eq (10). For $t>t_{\max } / 2$, evaporation occurs based on the droplet evaporation probability DEP by using Eq (13). It should be noted that for generating monolayer and droplet evaporation probability matrices, it is necessary to generate the correspondent substrate energy vector and contact angle vector by using Eqs (9) and (12) respectively.

Step 3: Generating random permutation based step size matrix

A random permutation based step size matrix is generated according to Eq. (14)

Step 4: Generating evaporated water molecules and updating the matrix of water molecules

The evaporated set of water molecules $\mathrm{WM}^{(\mathrm{t}+1)}$ is generated by adding the product of step size matrix and evaporation matrix to the current set of molecules $\mathrm{WM}^{(\mathrm{t})}$ by using Eq. (15). These molecules are evaluated 
based on the objective function. For the molecule $\mathrm{i}(\mathrm{i}=1,2, \ldots . \mathrm{nWM})$ if the newly generated molecule is better than the current one, the latter should be replaced. Return the best water molecule as the output of the algorithm Step 5: Terminating condition check

If the number of iteration of the algorithm $(\mathrm{t})$ becomes larger than the maximum number of iterations $\left(\mathrm{t}_{\max }\right)$, the algorithm terminates. Otherwise go to step 2.

The detailed flowchart for the implementation of WEO algorithm for DED problem is shown in Fig 1.

\section{Examples And Simulation Results}

The proposed methodology has been tested with three test systems and the proposed algorithm is developed in Matlab environment and is implemented using Intel(R) Core(TM) i5-4200U CPU@1.60 GHz 2.30 $\mathrm{GHz}$ processor. The effectiveness of the proposed WEO algorithm for ELD problem has been validated by comparing the simulation results obtained from the other method which is available in literature. The WEO algorithm parameters for all test systems are chosen as the number of water molecules $(\mathrm{nWM})=10$, maximum number of algorithm iteration $\left(\mathrm{t}_{\max }\right)=100, \mathrm{MEP}_{\min }=0.03, \mathrm{MEP}_{\max }=0.6, \mathrm{DEP}_{\min }=0.6, \mathrm{DEP}_{\max }=1$.

\subsection{Test System 1}

This test system consist of 5 generating units is consider to validate the proposed method. Here spinning reserve, valve point loading and transmission losses are included. The test system particulars are available in the literature [8]. The best generation schedule obtained by the proposed WEO algorithm for a 5 generating units system is given in the Table 1. From the simulation results it is clear that the proposed WEO algorithm meet the load demand for entire planning period of $24 \mathrm{hr}$ and obtain the minimized fuel cost of 422993.6318(\$) with a loss of 195.1953(MW) with satisfying system constraints power balance, generator power limit.

Table 1. The best generation schedule using WEO algorithm for 5 unit system

\begin{tabular}{|c|c|c|c|c|c|c|c|c|c|c|c|}
\hline Unit & Load & $\mathrm{P}_{1}(\mathrm{MW})$ & $\mathrm{P}_{2}(\mathrm{MW})$ & $\mathrm{P}_{3}(\mathrm{MW})$ & $\mathrm{P}_{4}(\mathrm{MW})$ & $P_{5}(\mathrm{MW})$ & $\mathrm{P}_{\text {loss }}(\mathrm{MW})$ & Generation & $\Delta_{t}^{(1)}$ & $\Delta_{\mathrm{t}}^{(2)}$ & $\Delta_{t}^{(3)}$ \\
\hline 1 & 410 & 20.6014 & 98.5423 & 30.0000 & 124.9100 & 139.7583 & 3.8120 & 1249.5795 & 490.6880 & 175.9577 & 26.5000 \\
\hline 2 & 435 & 10.0000 & 97.9621 & 66.4957 & 124.9048 & 139.7598 & 4.1224 & 1422.7021 & 464.1276 & 175.2879 & 26.0833 \\
\hline 3 & 475 & 10.0354 & 98.5257 & 106.4960 & 124.9517 & 139.7722 & 4.7810 & 1339.8280 & 421.4690 & 172.7243 & 25.4168 \\
\hline 4 & 530 & 10.0013 & 98.5810 & 112.7087 & 174.9513 & 139.7706 & 6.0129 & 1659.2123 & 362.4871 & 169.9190 & 24.5000 \\
\hline 5 & 558 & 10.0000 & 92.9923 & 112.6655 & 209.8147 & 139.9279 & 7.4004 & 1587.8947 & 331.6996 & 162.2853 & 24.0333 \\
\hline 6 & 608 & 10.0000 & 98.5409 & 112.6710 & 209.8153 & 184.9576 & 7.9848 & 1872.0504 & 278.6152 & 156.3338 & 23.2000 \\
\hline 7 & 626 & 10.0000 & 72.4515 & 112.6740 & 209.8158 & 229.5193 & 8.4606 & 1840.6093 & 259.2394 & 158.8842 & 22.9000 \\
\hline 8 & 654 & 12.7044 & 98.5437 & 112.6727 & 209.8160 & 229.5192 & 9.2560 & 1797.2305 & 229.0494 & 153.9403 & 22.4333 \\
\hline 9 & 690 & 42.7044 & 105.4542 & 112.6735 & 209.8160 & 229.5191 & 10.1672 & 2012.1289 & 190.3328 & 145.2300 & 21.8333 \\
\hline 10 & 704 & 64.0108 & 98.5398 & 112.6735 & 209.8158 & 229.5196 & 10.5595 & 1996.5951 & 175.2405 & 132.4336 & 21.6000 \\
\hline 11 & 720 & 75.0000 & 104.0359 & 112.6735 & 209.8158 & 229.5196 & 11.0448 & 2037.9302 & 157.9552 & 115.1483 & 16.3333 \\
\hline 12 & 740 & 75.0000 & 124.7111 & 112.6735 & 209.8158 & 229.5196 & 11.7200 & 2180.0222 & 136.2800 & 93.4731 & 11.2889 \\
\hline 13 & 704 & 64.0108 & 98.5398 & 112.6735 & 209.8158 & 229.5196 & 10.5595 & 1996.5951 & 175.2405 & 132.4336 & 21.6000 \\
\hline 14 & 690 & 49.6196 & 98.5398 & 112.6735 & 209.8158 & 229.5196 & 10.1683 & 1977.6613 & 190.3317 & 147.5248 & 21.8333 \\
\hline 15 & 654 & 19.6187 & 91.5860 & 112.6734 & 209.8158 & 229.5200 & 9.2139 & 1862.7466 & 229.0861 & 157.4842 & 22.4333 \\
\hline 16 & 580 & 10.0000 & 75.1565 & 112.6734 & 159.8087 & 229.5200 & 7.1886 & 1892.8463 & 308.8114 & 171.0000 & 23.6667 \\
\hline 17 & 558 & 10.0000 & 87.7145 & 112.6735 & 124.9078 & 229.5323 & 6.8290 & 1615.0520 & 332.2710 & 172.1000 & 24.0333 \\
\hline 18 & 608 & 10.0000 & 98.5403 & 112.6759 & 165.0898 & 229.5200 & 7.8260 & 1853.1315 & 278.7740 & 166.0597 & 23.2000 \\
\hline 19 & 654 & 12.7080 & 98.5407 & 112.6735 & 209.8160 & 229.5196 & 9.2578 & 1797.2251 & 229.0422 & 153.9433 & 22.4333 \\
\hline 20 & 704 & 42.7078 & 119.9405 & 112.6735 & 209.8158 & 229.5196 & 10.6572 & 2115.5135 & 175.1428 & 130.0437 & 21.6000 \\
\hline 21 & 680 & 39.3528 & 98.5399 & 112.6735 & 209.8158 & 229.5196 & 9.9016 & 1944.5975 & 201.0984 & 152.6443 & 22.0000 \\
\hline 22 & 605 & 10.0001 & 98.5399 & 112.6735 & 162.1377 & 229.5196 & 7.8708 & 1843.5182 & 281.8792 & 166.2101 & 23.2500 \\
\hline 23 & 527 & 10.0000 & 98.5398 & 112.6733 & 124.9081 & 186.7828 & 5.9040 & 1677.5493 & 365.7460 & 170.1102 & 24.5500 \\
\hline 24 & 463 & 10.0000 & 80.1559 & 112.6731 & 124.9082 & 139.7598 & 4.4970 & 1421.4122 & 434.3530 & 176.8500 & 25.6167 \\
\hline
\end{tabular}




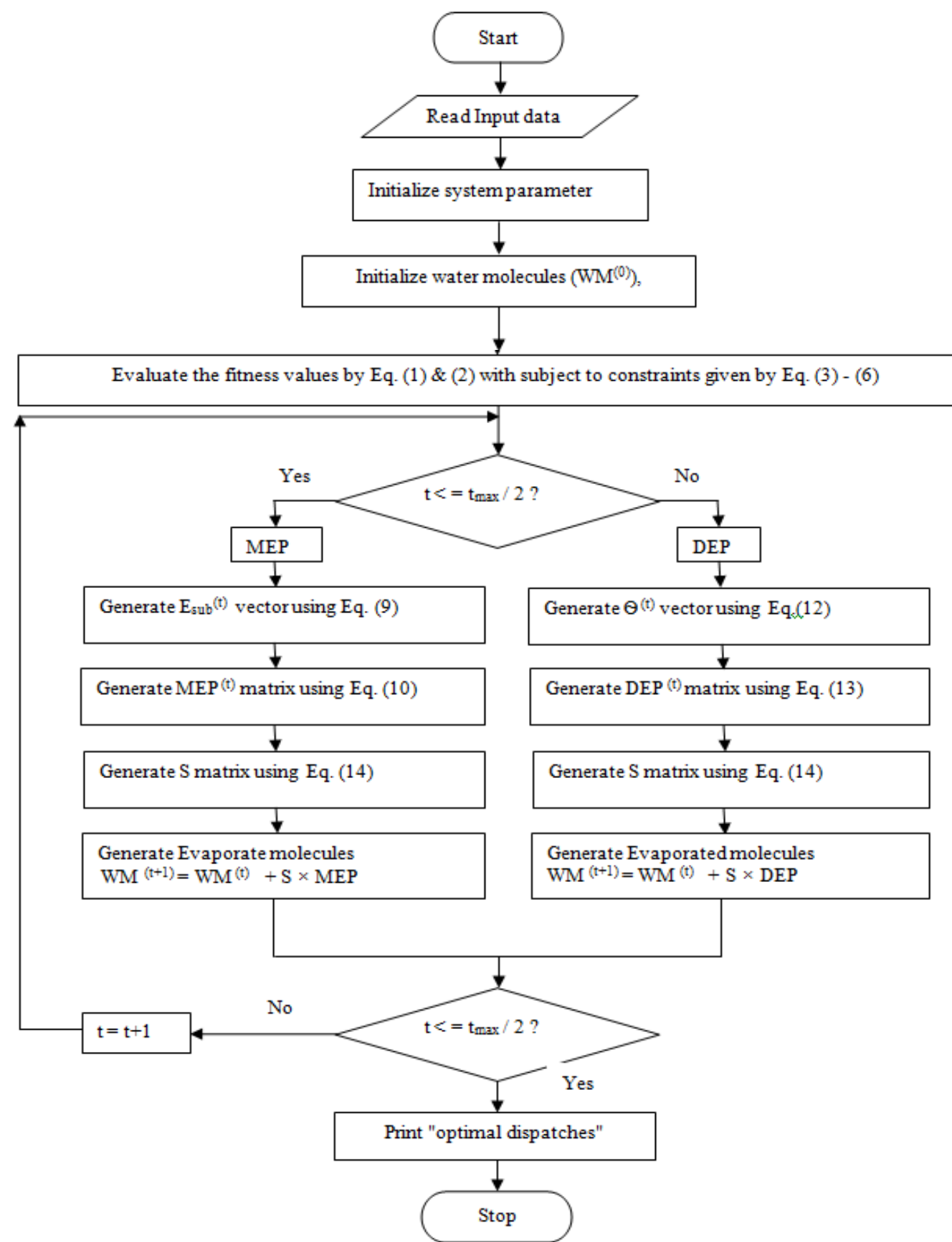

Fig. 1 Flowchart for the proposed WEO algorithm to solve DED

The results also imply that every hour the ramp rate limit has to be maintained. The comparison of best, worst and the mean value of the total fuel cost is presented in Table 2. The proposed WEO algorithm achieve the best cost of 42993.6318(\$), worst cost of 43089.63(\$), and mean cost of 43009.74(\$). From the comparison it is clear that the proposed algorithm achieve the best results in comparison with SA [8], APSO [28], AIS [9], TLA [19] and MTLA [19]. The cost convergence characteristic curve is depicting in Fig 2. The convergence curve demonstrate that the cost is converged from larger value to smaller value ensure that the WEO algorithm is capable of producing better results than existing algorithms.

The spinning reserve requirements are set to 5\% of load demand in each hour for this test system with time period of $24-$ hour. Here the SRRs are formulated in three different ways, $\Delta_{T}^{(1)}, \Delta_{T}^{(2)}, \Delta_{T}^{\left({ }^{(3)}\right.}$ and are computed by WEO algorithm is also presented in Table1. All the SRRs values are positive ensure that the proposed algorithm maintained sufficient SRR in each hour. 


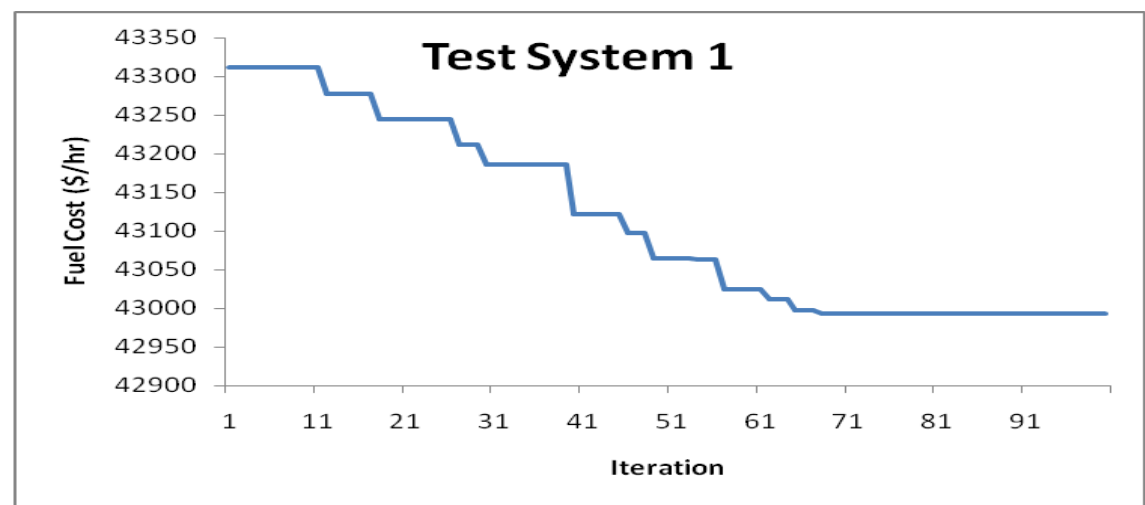

Fig. 2 Convergence curve of the Test system 1

Table 2. The best generation schedule using WEO algorithm for 5 unit system

\begin{tabular}{|l|l|l|l|l|}
\hline \multirow{2}{*}{ Solution Techniques } & \multicolumn{2}{|l|}{ Total Fuel cost (\$) } & \multirow{2}{*}{ Time (Min) } \\
\cline { 2 - 4 } & Best Value & Mean Value & Worst Value & \\
\hline SA[8] & 47356 & NA & NA & 4.395 \\
\hline APSO[28] & 44678 & NA & NA & NA \\
\hline AIS[9] & 44385.4 & 44758.8 & 45553.8 & 5.333 \\
\hline TLA[19] & 43132.9 & 43209.4 & 43897.4 & 0.060 \\
\hline MTLA[19] & 43048.4 & 43077.9 & 43128.5 & 0.071 \\
\hline WEO & $\mathbf{4 2 9 9 3 . 6 3 1 8}$ & $\mathbf{4 3 0 0 9 . 7 4}$ & $\mathbf{4 3 0 8 9 . 6 3}$ & $\mathbf{0 . 0 0 7 8}$ \\
\hline
\end{tabular}

NA: Not available in the literature

\subsection{Test System 2}

To demonstrate the validity of the proposed WEO algorithm a ten unit test system is considered with valve point loading effect and ramp rate limit. In this test system losses are neglected. The test system particulars are available in the literature [26]. The simulation results of 10 unit test system for a planning period of 24 hour obtained by the proposed WEO algorithm is presented in Table 3. The simulated results make sure that the proposed algorithm reaches the least cost with satisfying system constraints out and out. The comparison of total fuel cost in comparison with existing algorithms is presented in Table 4. It is evident from the comparison the proposed algorithm alone achieve the minimized cost than earlier reported algorithms. The objective value versus iteration curve is plotted in Fig. 3. From the convergence curve it is clear that the proposed WEO algorithm is efficient in handling system constraints and obtains the competitive results than existing algorithms.

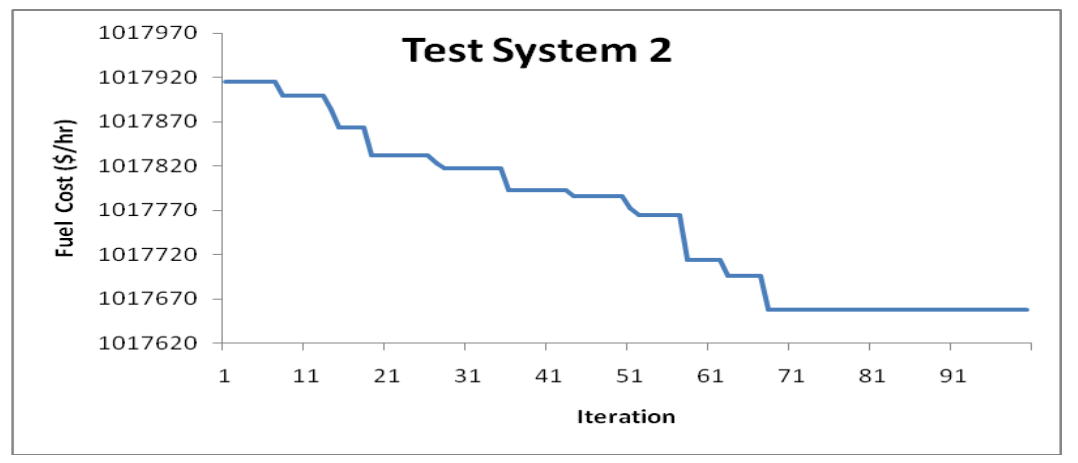

Fig. 3 Convergence curve of the Test system 2

\subsection{Test System 3}

In this case, the same 10 unit test system is considered along with network loss. The result obtained by the proposed WEO is present in table 5. The simulation results shows that the WEO algorithm meet the load demand in each hour and fulfill the system constraints. The proposed algorithm achieve the best optimal total cost of 1,038,313 \$ with total loss of $801.87 \mathrm{MW}$. The total fuel cost comparison of 10 unit test system with loss is presented in Table 6. The comparisons imply that proposed WEO algorithm alone reach the minimized cost than existing algorithms. The Fig. 4 shows the cost convergence curve of test system 3 . The convergence curves make clear that the results converged from larger values guarantee that the proposed WEO algorithm is efficient and obtain better results than earlier reported techniques. 


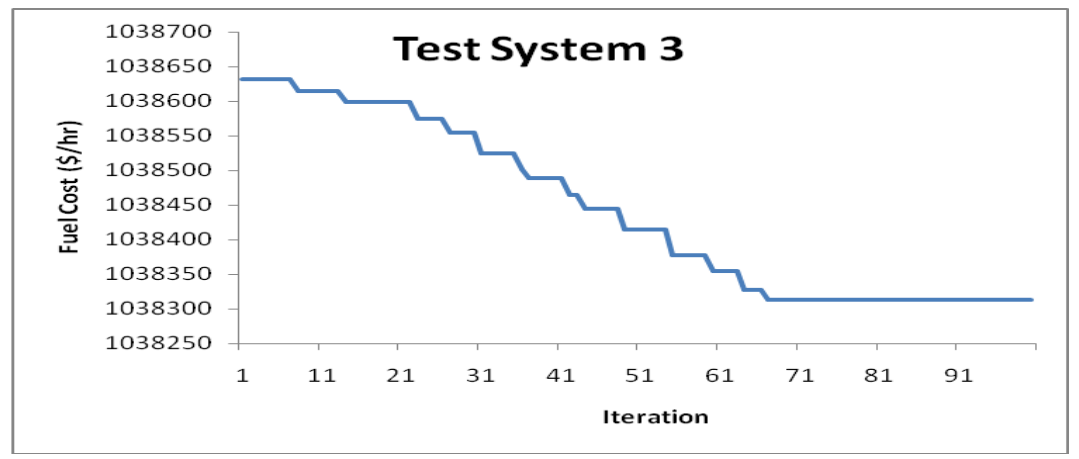

Fig 4: Convergence curve of the Test system 3

Table 3. The best generation schedule using WEO algorithm for 10 unit system without loss

\begin{tabular}{|c|c|c|c|c|c|c|c|c|c|c|c|c|}
\hline Hour & Load & $\mathbf{P}_{1}$ & $\mathbf{P}_{2}$ & $\mathbf{P}_{3}$ & $\mathbf{P}_{4}$ & $\mathbf{P}_{5}$ & $P_{6}$ & $\mathbf{P}_{7}$ & $\mathbf{P}_{8}$ & $\mathbf{P}_{9}$ & $\mathbf{P}_{10}$ & Cost (\$) \\
\hline 1 & 1036 & 150 & 135 & 194.08 & 60 & 122.87 & 122.46 & 129.59 & 47 & 20 & 50 & 28238.48 \\
\hline 2 & 1110 & 150 & 135 & 268.08 & 60 & 122.87 & 122.46 & 129.59 & 47 & 20 & 55 & 29946.84 \\
\hline 3 & 1258 & 226.63 & 215 & 309.32 & 60 & 73 & 122.46 & 129.59 & 47 & 20 & 55 & 33123.67 \\
\hline 4 & 1406 & 303.26 & 222.28 & 323.56 & 60 & 122.85 & 122.46 & 129.59 & 47 & 20 & 55 & 36290.84 \\
\hline 5 & 1480 & 379.87 & 302.27 & 290.82 & 60 & 73.00 & 122.45 & 129.59 & 47 & 20 & 55 & 37903.48 \\
\hline 6 & 1628 & 456.50 & 309.53 & 305.06 & 60 & 122.87 & 122.45 & 129.59 & 47 & 20 & 55 & 40981.77 \\
\hline 7 & 1702 & 456.50 & 309.53 & 308.06 & 80 & 172.73 & 123.58 & 129.59 & 47 & 20 & 55 & 42902.12 \\
\hline 8 & 1776 & 456.50 & 309.53 & 308.06 & 126 & 172.73 & 151.58 & 129.59 & 47 & 20 & 55 & 44651.6 \\
\hline 9 & 1924 & 456.50 & 389.54 & 305.34 & 176 & 222.60 & 122.43 & 129.59 & 47 & 20 & 55 & 47903.64 \\
\hline 10 & 2072 & 456.50 & 396.80 & 298.50 & 226.01 & 222.60 & 160 & 129.59 & 47 & 50 & 55 & 51808.93 \\
\hline 11 & 2146 & 456.50 & 396.80 & 340 & 248.13 & 122.63 & 160 & 129.59 & 85.29 & 52.06 & 55 & 53407.02 \\
\hline 12 & 2220 & 456.50 & 460.00 & 300.80 & 298.14 & 222.60 & 160 & 129.59 & 85.31 & 52.06 & 55 & 55306.5 \\
\hline 13 & 2072 & 456.50 & 396.80 & 297.40 & 248.14 & 222.60 & 158.60 & 129.59 & 85.31 & 22.06 & 55 & 51415.72 \\
\hline 14 & 1924 & 456.50 & 396.80 & 287.47 & 198.14 & 172.73 & 122.45 & 129.59 & 85.31 & 20 & 55 & 48041.52 \\
\hline 15 & 1776 & 379.88 & 396.80 & 283.27 & 180.82 & 122.86 & 122.45 & 129.59 & 85.31 & 20 & 55 & 44594.07 \\
\hline 16 & 1554 & 302.88 & 396.80 & 283.27 & 180.82 & 122.86 & 122.45 & 129.59 & 85.31 & 20 & 55 & 39969.23 \\
\hline 17 & 1480 & 222.62 & 309.53 & 288.21 & 120.42 & 122.81 & 122.45 & 129.59 & 85.31 & 20 & 55 & 37973.28 \\
\hline 18 & 1628 & 303.25 & 316.80 & 317.79 & 130.83 & 73 & 122.45 & 129.59 & 85.31 & 20 & 55 & 41215.03 \\
\hline 19 & 1776 & 379.87 & 389.53 & 301.09 & 120.42 & 172.73 & 122.45 & 129.59 & 85.31 & 20 & 55 & 44510.52 \\
\hline 20 & 2072 & 456.50 & 460.00 & 312.59 & 170.42 & 222.60 & 160.00 & 129.59 & 85.31 & 20 & 55 & 51768.67 \\
\hline 21 & 1924 & 456.50 & 396.80 & 315.33 & 120.42 & 222.60 & 122.45 & 129.59 & 85.31 & 20 & 55 & 47708.92 \\
\hline 22 & 1628 & 379.87 & 316.80 & 275.83 & 70.42 & 172.73 & 122.45 & 129.59 & 85.31 & 20 & 55 & 41496.69 \\
\hline 23 & 1332 & 303.23 & 236.80 & 196.74 & 60 & 122.88 & 122.45 & 129.59 & 85.31 & 20 & 55 & 35037.12 \\
\hline 24 & 1184 & 226.61 & 222.26 & 189.78 & 60 & 73 & 122.45 & 129.59 & 85.31 & 20 & 55 & 31461.86 \\
\hline
\end{tabular}

Total Cost

$1,017,657.52$

Table 4. Comparison of total fuel cost for 10 unit system without loss

\begin{tabular}{|c|c|c|c|c|}
\hline \multirow{2}{*}{ Method } & \multicolumn{3}{|c|}{ Total Fuel cost (\$) } & \multirow{2}{*}{ Time (min) } \\
\hline & BEST & MEAN & WORST & \\
\hline SQP [10] & $1,051,163$ & NA & NA & 0.421 \\
\hline EP [10] & $1,048,638$ & NA & NA & 15.049 \\
\hline HS [12] & $1,046,726$ & NA & NA & NA \\
\hline DE [11] & $1,036,756$ & $1,040,586$ & $1,452,558$ & 0.20 \\
\hline GA [7] & $1,033,481$ & $1,038,014$ & $1,042,606$ & NA \\
\hline SOA [15] & $1,023,946$ & $1,026,289$ & $1,029,213$ & NA \\
\hline AIS [9] & $1,021,980$ & $1,023,156$ & $1,024,973$ & 25.346 \\
\hline $\mathrm{ABC}[13]$ & $1,021,576$ & $1,022,686$ & $1,024,316$ & 2.603 \\
\hline TLA [16] & $1,019,925$ & $1,020,411$ & $1,021,118$ & 0.049 \\
\hline ICA [14] & $1,018,467$ & $1,019,291$ & $1,021,796$ & $\mathrm{NA}$ \\
\hline HDE [29] & $1,031,077$ & NA & NA & NA \\
\hline IPSO [17] & $1,023,807$ & $1,026,863$ & NA & 0.060 \\
\hline
\end{tabular}


Water Evaporation Optimization Algorithm for Solving Dynamic Economic Dispatch

\begin{tabular}{|l|l|l|l|l|}
\hline CDBCO [30] & $1,021,500$ & $1,024,300$ & NA & 0.67 \\
\hline CDE [18] & $1,019,123$ & $1,020,870$ & $1,023,115$ & 0.32 \\
\hline ICPSO [31] & $1,019,072$ & $1,020,027$ & NA & 0.350 \\
\hline CSAPSO [32] & $1,018,767$ & $1,019,874$ & NA & 0.350 \\
\hline EAPSO [33] & $1,018,510$ & $1,018,710$ & $1,019,302$ & 0.625 \\
\hline TVAC-IPSO [34] & $1,018,217$ & $1,018,965$ & $1,020,417$ & 2.718 \\
\hline EBSO [35] & $1,017,147$ & $1,017,526$ & $1,017,891$ & 0.205 \\
\hline HQPSO [36] & $1,031,559$ & $1,033,837$ & $1,036,681$ & 0.773 \\
\hline AIS-SQP [23] & $1,029,900$ & NA & NA & NA \\
\hline DGPSO [37] & $1,028,835$ & $1,030,183$ & NA & 4.809 \\
\hline ECE [25] & $1,022,271$ & $1,023,334$ & NA & 0.329 \\
\hline SOA-SQP [15] & $1,021,460$ & $1,023,840$ & $1,026,852$ & NA \\
\hline AHDE [38] & $1,020,082$ & $1,022,476$ & NA & 1.10 \\
\hline HHS [12] & $1,019,091$ & $\mathbf{N A}$ & $1,022,284$ & 10.194 \\
\hline HIGA [39] & $1,018,473$ & $1,019,328$ & $1,019,286$ & 3.53 \\
\hline CSO[26] & $1,017,660$ & $1,018,120$ & $\mathbf{1 , 0 1 9 , 1 1 6}$ & 0.961 \\
\hline WEO & $\mathbf{1 , 0 1 7 , 6 5 7 . 5 2}$ & $\mathbf{1 , 0 1 8 , 1 0 9}$ & $\mathbf{1 . 1 3 0}$ \\
\hline
\end{tabular}

Table 5. The best generation schedule using WEO algorithm for 10 unit system with loss

\begin{tabular}{|c|c|c|c|c|c|c|c|c|c|c|c|c|c|}
\hline Hour & Load & $\mathbf{P}_{1}$ & $\mathbf{P}_{2}$ & $\mathbf{P}_{3}$ & $\mathbf{P}_{4}$ & $\mathbf{P}_{5}$ & $\mathbf{P}_{6}$ & $\mathbf{P}_{7}$ & $\mathbf{P}_{8}$ & $\mathbf{P}_{9}$ & $\mathbf{P}_{10}$ & $\begin{array}{c}\text { Loss } \\
\text { (MW) }\end{array}$ & Cost (\$) \\
\hline 1 & 1036 & 150 & 135 & 206.21 & 60 & 122.88 & 122.45 & 129.59 & 47 & 20 & 55 & 12.13 & 28591.83 \\
\hline 2 & 1110 & 150 & 135 & 282.76 & 60 & 122.87 & 122.45 & 129.59 & 47 & 20 & 55 & 14.67 & 30148.99 \\
\hline 3 & 1258 & 226.62 & 135 & 307.50 & 60 & 172.73 & 122.45 & 129.59 & 47 & 20 & 55 & 17.89 & 33388.38 \\
\hline 4 & 1406 & 303.25 & 215.00 & 304.38 & 60 & 172.73 & 122.45 & 129.59 & 47 & 20 & 55 & 23.40 & 36824.88 \\
\hline 5 & 1480 & 379.87 & 222.27 & 297.40 & 60 & 172.73 & 122.45 & 129.59 & 47 & 20 & 55 & 26.31 & 38293.21 \\
\hline 6 & 1628 & 456.50 & 222.27 & 305.09 & 80 & 222.60 & 122.45 & 129.59 & 47 & 20 & 55 & 32.50 & 42004.14 \\
\hline 7 & 1702 & 456.50 & 302.27 & 312.13 & 120.42 & 172.73 & 122.45 & 129.59 & 47 & 20 & 55 & 36.08 & 43731.35 \\
\hline 8 & 1776 & 456.50 & 309.53 & 299.42 & 170.42 & 172.73 & 122.45 & 129.59 & 77 & 20 & 55 & 36.64 & 45622.45 \\
\hline 9 & 1924 & 456.50 & 389.53 & 299.42 & 189.55 & 222.60 & 122.45 & 129.59 & 85.31 & 20 & 55 & 45.95 & 49017.74 \\
\hline 10 & 2072 & 456.50 & 396.80 & 325.60 & 239.51 & 222.60 & 160 & 129.59 & 115.31 & 20 & 55 & 48.91 & 52817.04 \\
\hline 11 & 2146 & 458.45 & 396.78 & 340 & 239.51 & 222.60 & 160 & 129.59 & 120 & 20 & 55 & 50.93 & 54809.25 \\
\hline 12 & 2220 & 456.50 & 459.97 & 325.05 & 300 & 222.60 & 160 & 129.59 & 120 & 50 & 55 & 58.71 & 56961.01 \\
\hline 13 & 2072 & 456.50 & 396.76 & 298.44 & 300 & 222.60 & 122.44 & 129.59 & 85.30 & 20 & 55 & 49.33 & 52515.69 \\
\hline 14 & 1924 & 456.50 & 316.80 & 302.34 & 250.00 & 222.60 & 122.45 & 129.59 & 90 & 20 & 55 & 41.28 & 49246.06 \\
\hline 15 & 1776 & 379.86 & 309.51 & 294.61 & 241.18 & 172.72 & 122.45 & 129.59 & 85.31 & 20 & 55 & 34.22 & 45409.30 \\
\hline 16 & 1554 & 303.24 & 229.52 & 318.36 & 191.15 & 122.84 & 122.45 & 129.59 & 85.31 & 20 & 55 & 23.46 & 40658.66 \\
\hline 17 & 1480 & 226.62 & 222.26 & 287.67 & 180.79 & 172.73 & 122.44 & 129.59 & 85.31 & 20 & 55 & 22.42 & 38646.77 \\
\hline 18 & 1628 & 303.25 & 222.27 & 312.74 & 180.83 & 222.60 & 123.35 & 129.59 & 85.31 & 20 & 55 & 26.93 & 42017.57 \\
\hline 19 & 1776 & 379.84 & 302.27 & 313 & 180.83 & 222.60 & 122.44 & 129.59 & 85.31 & 20 & 55 & 34.88 & 45538.72 \\
\hline 20 & 2072 & 456.50 & 382.21 & 340 & 230.83 & 230.44 & 160 & 129.59 & 115.30 & 20 & 55 & 47.87 & 53382.49 \\
\hline 21 & 1924 & 456.50 & 396.80 & 301.35 & 180.83 & 222.60 & 122.45 & 129.59 & 85.31 & 20 & 55 & 46.42 & 48752.43 \\
\hline 22 & 1628 & 379.86 & 316.81 & 278.30 & 130.84 & 172.69 & 122.45 & 129.59 & 55.29 & 20 & 55 & 32.83 & 42479.09 \\
\hline 23 & 1332 & 303.25 & 236.80 & 198.33 & 118.34 & 122.87 & 122.45 & 129.59 & 47 & 20 & 55 & 21.20 & 35599.61 \\
\hline 24 & 1184 & 226.60 & 222.24 & 184.62 & 120.41 & 73 & 122.45 & 129.59 & 47 & 20 & 55 & 16.91 & 31856.02 \\
\hline
\end{tabular}

$801.87 \quad 1,038,313.49$

Table 6. The total fuel cost comparison of 10 unit system with loss

\begin{tabular}{|l|l|l|l|l|l|}
\hline \multirow{2}{*}{ Method } & \multicolumn{3}{|c|}{ Total Fuel cost (\$) } & \multirow{2}{*}{ Loss (MW) } & \multirow{2}{*}{ Time (min) } \\
\cline { 2 - 4 } & BEST & MEAN & WORST & NA & NA \\
\hline MIQP [6] & $1,038,550$ & NA & NA & NA & 47.23 \\
\hline EP [10] & $1,054,685$ & $1,057,323$ & NA & NA & 3.444 \\
\hline GA [7] & $1,052,251$ & $1,058,041$ & $1,062,511$ & 835.62 & 30.973 \\
\hline AIS [9] & $1,045,715$ & $1,047,050$ & $1,048,431$ & 817.80 & 3.408 \\
\hline ABC [13] & $1,043,381$ & $1,044,963$ & $1,046,805$ & 848.797 & NA \\
\hline ICA [14] & $1,040,758$ & $1,041,664$ & $1,043,173$ & NA & 0.180 \\
\hline IPSO [17] & $1,046,275$ & $1,048,154$ & NA & 839.31 & 1.53 \\
\hline CDBCO [30] & $1,042,900$ & $1,044,700$ & NA & 854.033 & 3.155 \\
\hline TVAC-IPSO [34] & $1,041,066$ & $1,042,118$ & $1,043,626$ & NA & 0.22 \\
\hline EBSO [35] & $1,038,915$ & $1,039,188$ & $1,039,272$ & NA & 27.53 \\
\hline EP-SQP [10] & $1,052,668$ & $1,053,771$ & NA & NA & 24.33 \\
\hline MHEP-SQP [10] & $1,050,054$ & $1,052,349$ & NA & NA & 5.99 \\
\hline DGPSO [37] & $1,049,167$ & $1,051,725$ & NA & & \\
\hline
\end{tabular}


Water Evaporation Optimization Algorithm for Solving Dynamic Economic Dispatch

\begin{tabular}{|l|l|l|l|l|l|}
\hline ECE [25] & $1,043,989$ & $1,044,470$ & NA & NA & 0.644 \\
\hline HIGA [39] & $1,041,088$ & $1,042,980$ & $1,044,927$ & 853.53 & 3.8 \\
\hline CSO[26] & $1,038,320$ & $1,039,374$ & $1,042,518$ & 802.62 & 1.481 \\
\hline Proposed WEO & $\mathbf{1 , 0 3 8 , 3 1 3}$ & $\mathbf{1 , 0 3 9 , 0 0 3}$ & $\mathbf{1 , 0 4 2 , 1 8 6}$ & $\mathbf{8 0 1 . 8 7}$ & $\mathbf{1 . 7 6 4}$ \\
\hline
\end{tabular}

\section{Conclusion}

A heuristic optimization method called WEO was developed for the purpose of optimal solution for the DED problem. The practical operational constraints of generators such as spinning reserve, ramp rate limits and valve point effect along with transmission loss were considered in the analysis. The feasibility and efficiency of the proposed method were demonstrated on five and ten unit test systems. The numerical results were compared with the recent optimization approaches. The numerical results revealed that the dispatch solution obtained by the proposed WEO approach led to a smaller operating cost than those found by other methods, which showed the capability of the algorithm to determine the global or near global solutions for the DED problem.

\section{References}

[1]. J. Wood, and B. F. Wollenberg, Power generation, operation and control, Second Edition. John Wiley and Sons. New York, 1996.

[2]. B. Somuah, and N. Khunaizi, Application of linear programming dispatch technique to dynamic generation allocation, IEEE Transaction Power System, 5(1), 1990, 20-26.

[3]. Chen, Non-convex economic dispatch: A direct search approach, Energy Conversion Management, 48 (1), $2007,219-225$.

[4]. L. G. Papageorgiou, and E. S. Fraga, A mixed integer quadratic programming formulation for the economic dispatch of generators with prohibited operating zones, Electrical Power and Energy System, 77, 2007, 1292-1296.

[5]. S. Hemamalini, and S. P. Simon, Dynamic economic dispatch using Maclaurin series based Lagrangian method, Energy Conversion Management, 51, 2010, 2122-2129.

[6]. M. Wang, HB. Gooi, SX. Chen, and S. Lu, A mixed integer quadratic programming for dynamic economic dispatch with valve point effect, IEEE Transaction Power System, 29(5), 2014, 2097-2106.

[7]. F. Li, R. Morgan, and D. Williams, Hybrid genetic approaches to ramping rate constrained dynamic economic dispatch, Electrical Power and Energy System, 43(2), 1997, 97-103.

[8]. M. Basu, C. K. Panigrahi, R. N. Chakrabarti, and P. K. Chattopadhyay, Simulated annealing technique for dynamic economic dispatch, Electrical Power Components and System, 34(5), 2006, 577-586.

[9]. S. Hemamalini, and S. P. Simon, Dynamic economic dispatch using artificial immune system for units with valve-point effect, International Journal Electrical Power and Energy System, 33(4), 2011, 868-874.

[10]. P. Attaviriyanupap, H. Kita, E. Tanaka, and J. Hasegawa, A hybrid EP and SQP for dynamic economic dispatch with nonsmooth fuel cost function, IEEE Transaction power system, 17(4), 2002, 411-416.

[11]. S. Subramanian and R. Balamurugan, Differential evolution-based dynamic economic dispatch of generating units with valve-point effects, Electrical Power Components and System, 36(8), 2008, 828-843.

[12]. VR. Pandi, and BK. Panigrahi, Dynamic economic load dispatch using hybrid swarm intelligence based harmony search algorithm, Expert System with Applications, 38(7), 2011, 8509-8514.

[13]. S. Hemamalini, and SP. Simon, Dynamic economic dispatch using artificial bee colony algorithm for units with valve-point effect, European Transactions of Electrical Power, 21(1), 2011, 70-81.

[14]. B. Mohammadi-Ivatloo, A. Rabiee, A. Soroudi, and M. Ehsan, Imperialist competitive algorithm for solving non-convex dynamic economic power dispatch, Energy, 44(1), 2012, 228-240.

[15]. S. Sivasubramani, and KS. Swarup, Hybrid SOA-SQP algorithm for dynamic economic dispatch with valve-point effects, Energy, 35(12), 2010, 5031-5036.

[16]. S. Banerjee, D. Maity, and CK. Chanda, Teaching learning based optimization for economic load dispatch problem considering valve point loading effect, International Journal of Electrical Power and Energy System,73, 2015, 456-464.

[17]. J. Cai, X. Ma, L. Li, and P. Hai, Chaotic particle swarm optimization for economic dispatch considering the generator constraints, Energy Conversion Management, 48(2), 2007, 654-663.

[18]. Y. Lu, J. Zhou, H. Qin, Y. Wang, and Y. Zhang, Chaotic differential evolution methods for dynamic economic dispatch with valvepoint effects, Engineering Applications of Artificial Intelligence, 24(2), 2011, 378-387.

[19]. Niknam Taher, Azizipanah-Abarghooee Rasou, and Aghaei Jamshid, A new modified teaching-learning algorithm for reserve constrained dynamic economic dispatch, IEEE Transaction Power System, 28, 2013, 749-763.

[20]. Niknam Taher, Azizipanah-Abarghooee Rasou, and Roosta Alireza, Reserve constrained dynamic economic dispatch: a new fast self-adaptive modified firefly algorithm, IEEE System Journal, 6, 2012, 635-646.

[21]. JS. Alsumait, M. Qasem, JK. Sykulski, and AK. Al-Othman, An improved pattern search based algorithm to solve the dynamic economic dispatch problem with valve-point effect, Energy Conversation Management, 51(10), 2010, 2062-2067.

[22]. Mohammadi-Ivatloo Behnam, Rabiee Abbas, and Soroudi Alireza, Nonconvex dynamic economic power dispatch problems solution using hybrid immune-genetic algorithm, IEEE System Journal, 7(4), 2013,777-785.

[23]. M. Basu, Hybridization of artificial immune systems and sequential quadratic programming for dynamic economic dispatch, Electrical Power Components and System, 37(9), 2009, 1036-1045.

[24]. T. Aruldoss Albert Victoire, and A. Ebenezer Jeyakumar, A modified hybrid EP-SQP approach for dynamic dispatch with valvepoint effect, Electrical Power and Energy System, 27, 2005, 594-601.

[25]. Immanuel Selvakumar, Enhanced cross-entropy method for dynamic economic dispatch with valve-point effects, Electrical Power and Energy System, 33(3), 2011, 783-790.

[26]. A. Meng, H. Hu, H. Yin, X. Peng, and Z. Guo, Crisscross optimization algorithm for large scale dynamic economic dispatch problem with valve point effects, Energy, 93, 2015, 2175-2190.

[27]. A. Kaveh, and T. Bakhshpoori, Water Evaporation Optimization: A novel physically inspired optimization algorithm, Computer and Structures, 167, 2016, 69-85.

[28]. K. Panigrahi, and V. R. Pandi, and S. Das, Adaptive particle swarm optimization approach for static and dynamic economic load dispatch, Energy Conversation Management, 49(6), 2008, 1407-1415.

[29]. X. Yuan, L. Wang, Y. Zhang, and Y. Yuan, A hybrid differential evolution method for dynamic economic dispatch with valve-point effects, Expert Systems and Applications, 36(2), 2009, 4042-4048. 
[30]. P. Lu, J. Zhou, H. Zhang, R. Zhang, and C. Wang, Chaotic differential bee colony optimization algorithm for dynamic economic dispatch problem with valve-point effects, Electrical Power and Energy System, 62(11), 2014, 130-143.

[31]. Y. Wang, J. Zhou, H. Qin, and Y. Lu, Improved chaotic particle swarm optimization algorithm for dynamic economic dispatch problem with valve-point effects, Energy Conversation Management, 51(12), 2010, 2893-2900.

[32]. Y. Wang, J. Zhou, Y. Lu, and H. Qin, Chaotic self-adaptive particle swarm optimization algorithm for dynamic economic dispatch problem with valve-point effects, Expert System Application, 38, 2011, 4231-4237.

[33]. T. Niknam, and F. Golestaneh, Enhanced adaptive particle swarm optimization algorithm for dynamic economic dispatch of units considering valve-point effects and ramp rates, IET Generation Transmission Distribution, 6(5), 2012, 424-4325.

[34]. Mohammadi-Ivatloo, A. Rabiee, and M. Ehsan, Time-varying acceleration coefficients IPSO for solving dynamic economic dispatch with non-smooth cost function, Energy Conversation Management, 56(4), 2012, 175-183.

[35]. Niknam Taher, and Golestaneh Faranak. Enhanced bee swarm optimization algorithm for dynamic economic dispatch, IEEE Systems Journal, 7, 2013, 754-762.

[36]. S. Chakraborty, T. Senjyu, A. Yona, AY. Saber, and T. Funabashi, Solving economic load dispatch problem with valve-point effects using a hybrid quantum mechanics inspired particle swarm optimization, IET Generation Transmission Distribution, 5(10), 2011, 1042-1052.

[37]. T. Aruldoss Albert Victoire, and A. Ebenezer Jeyakumar, Deterministically guided PSO for dynamic dispatch considering valvepoint effect, Electric Power System Reasearch, 73, 2005, 313-322.

[38]. Y. Lu, J. Zhou, H. Qin, Y. Li, and Y. Zhang, An adaptive hybrid differential evolution algorithm for dynamic economic dispatch with valve-point effects, Expert Systems Application, 37 (7), 2010, 484-489.

[39]. Mohammadi-Ivatloo Behnam, and Rabiee Abbas, Soroudi Alireza, Nonconvex dynamic economic power dispatch problems solution using hybrid immune-genetic algorithm, IEEE Systems Journal, 7(4), 2013, 777-785. 